\title{
43. COMMISSION DE LA MAGNE'TO-HYDRODYNAMIQUE ET DE LA PHYSIQUE DES GAZ IONISES
}

President: H. Alfvén.

\section{Report of Meeting, 77 August I96r}

Secretary: C.-G. Fälthammar.

\section{Business meeting}

The Executive Committee had made proposals for President and Vice-President and for members of the Organizing Committee, as follows:

President: H. Alfvén

Vice-President: L. Spitzer

Organizing Committee: L. Biermann, T. G. Cowling, and A. B. Severny.

The meeting had no objections to these proposals.

Commission 43, which was formally established at the General Assembly in Moscow, I958, was meeting for the first time, and the President emphasised that the present Draft Report represented his personal view of what should be the scope of the Commission. He asked for comments on the report and suggestions for future changes in the scope. After a brief discussion whether or not ionospheric phenomena should be included, it was agreed not to make any recommendations for changes at present. Due to the rapid progress in the various fields of research involved, the possibility should be left open for future changes that might become desirable by forthcoming developments in any of these fields of research. S. Chapman suggested some minor changes in the Draft Report.

\section{Scientific meeting}

A second session of the meeting was devoted to a discussion on "Electro-magnetic Conditions in Interplanetary Space". Invited contributions were given by E. R. Mustel on "Scientific Results of U.S.S.R. Space-Probe Measurements" and by C. P. Sonett on "Scientific Results of U.S. Space-Probe Measurements".

Short communications were also presented by T. Gold on the escape of particles from the Earth's radiation belts and by $L$. Mestel on the equatorial acceleration in the Sun.

EXPERIMENTS ON PLASMA

AND MAGNETIC FIELD INVESTIGATIONS BY MEANS OF SOVIET SPACE PROBES

\section{E. R. Mustel}

The purpose of this paper is to give a brief review of some results of experiments, carried out by Soviet space probes, aimed at investigating plasma and magnetic fields.

I. In I958 a group of specialists in radiophysics under the leadership of Dr K. I. Gringauz used spherical probes screened by spherical grids, the so called ion traps $(\mathbf{I}, \mathbf{2})$, to investigate the concentration of positive ions in the ionosphere with Sputnik III. Later, traps of charged particles were placed on all containers with scientific instruments jettisoned from Soviet cosmic rockets (starting from the first artificial planet launched on 2 January 1959 and including the automatic interplanetary station launched to Venus on 12 February 1961). The third electrode-an internal grid-has been introduced in these traps to suppress photo- 
emissions from the collector, which are produced by ultra-violet radiation from the Sun, and limit sensitivity of the device to the stream of charged particles coming into the trap from the surrounding medium (Fig. I).

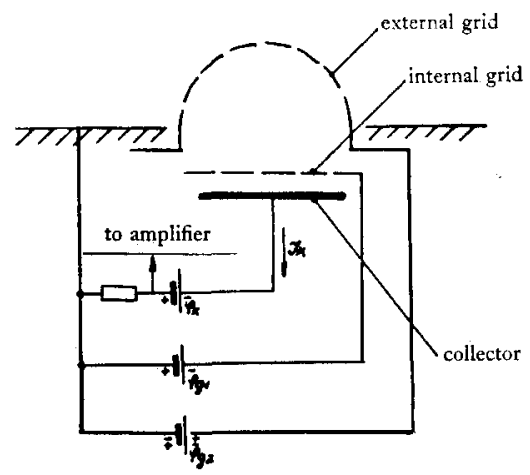

Fig. I. Schematic diagram of ion trap, showing the internal grid.

There is no possibility in this communication of describing the details of the methods of measurement by the traps of charged particles on each space probe (changed after each experiment); nor to describe the theoretical considerations on the basis of which measured currents in the collector circuits of the traps were reduced to concentrations of charged particles. This material has been published in Soviet scientific periodicals $(3,4,5)$ and was presented by Gringauz in April $196 \mathrm{r}$ in Florence at the Second International Space Science Symposium (6). Below are given the principal conclusions, made on the basis of experimental data obtained by those scientists who carried out the experiments. A group of specialists in astrophysics, under the leadership of I. S. Shklovsky, took part in the interpretation of some results obtained within the geomagnetic field at distances less than $100000 \mathrm{~km}$ from the Earth.

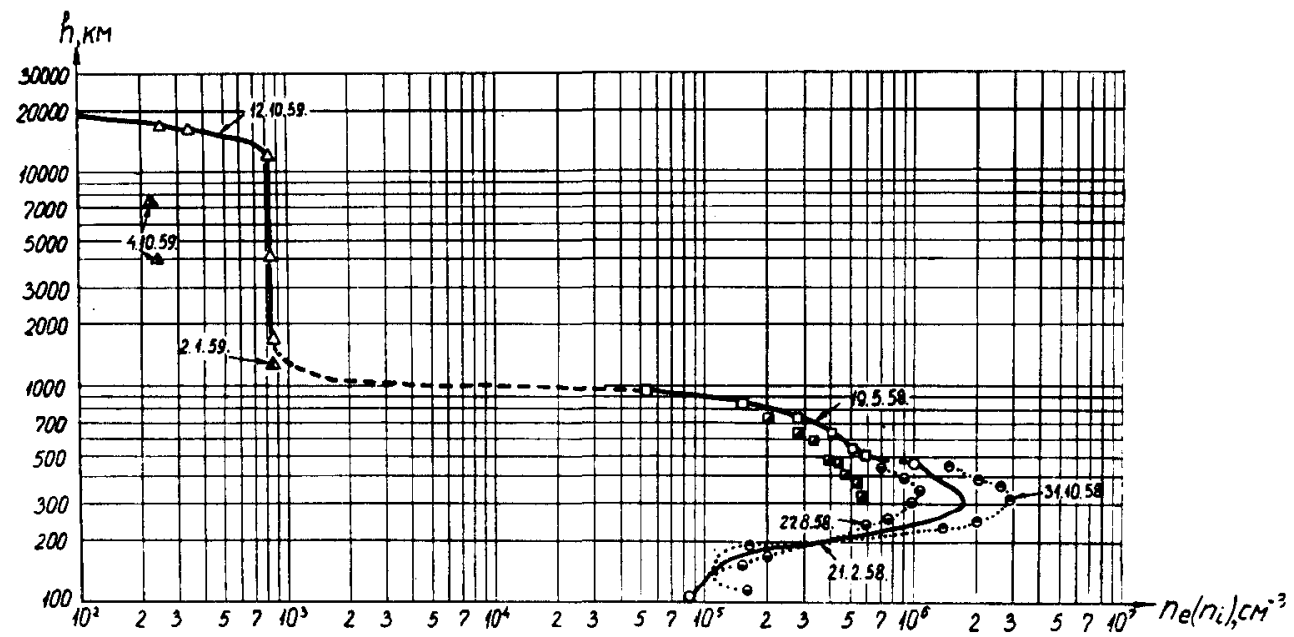

Fig. 2. Showing the variation of concentration of charged particles near the Earth--see text. 
Fig. 2 shows the tentative variation of the concentration of charged particles near the Earth for the daylight period of time close to the maximum of solar activity (based on Soviet experiments in 1958-9 made with geophysical rockets, on Sputnik III and space probes). Corresponding dates are indicated near experimental points; a dotted part of the curve indicates an area in which there are no experimental data.

Treatment of the results in measuring collector currents in traps of charged particles, placed on the first cosmic rockets, showed that at altitudes of about 50000 to $75000 \mathrm{~km}$ above the Earth, beyond the limits of the radiation belt, there have been registered electron fluxes of about $10^{8}$ per $\mathrm{cm}^{2}$ per sec and with energies exceeding $200 \mathrm{ev}$ (3). At the same time the energies of these electrons are insufficient to be registered with the help of counters of cosmic rays which had registered electrons of the outer radiation belt with the same rockets.

These results gave reasons to draw conclusions about the existence, beyond the limits of two radiation belts, of a third outermost belt of charged particles, the streams of which are larger than those in the second radiation belt, but the energy of which is less $(4,5)$. It was supposed, as had been done in the case of radiation belts, that the boundaries of that outermost belt of charged particles are situated along geomagnetic lines of force. The location of radiation belts ( $I$ and 2 ) and the outermost belt of charged particles (3) is shown in Fig 3 .

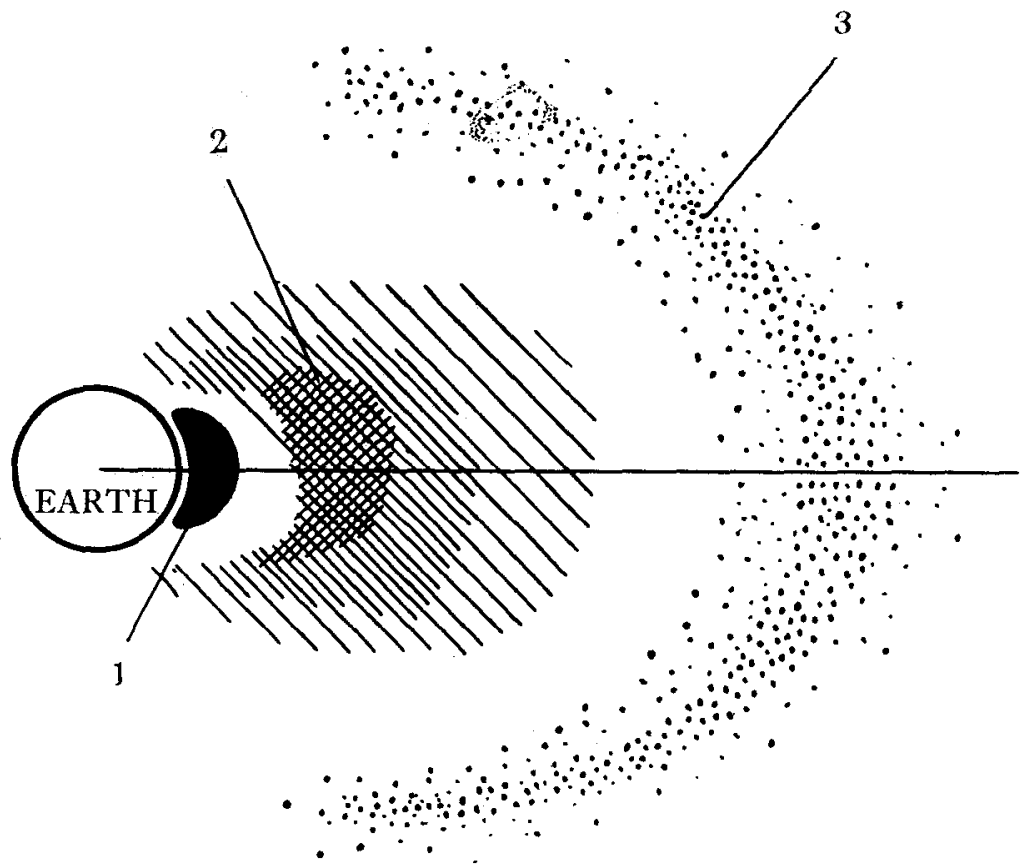

Fig. 3. Radiation belts ( $I$ and 2) and belts of charged particles (3) round the Earth.

After launching the American space probe Pioneer V in March, I960, there were published the results of measurements of the geomagnetic field carried out with it by Dr C. P. Sonett and other scientists (7). Deviations of the measured magnitudes of the geomagnetic field from the theoretical magnitudes of the dipole field, led American scientists to conclude the existence of a current-ring surrounding the Earth. A comparison of Sonett's data (8) with those published earlier by Gringauz and others $(3,4)$ showed that the current-ring, computed according to 
magnetic measurements, and the streams of electrons, revealed in Soviet experiments with three-electrode traps, are placed in the same region of space (8).

Thus, the above-mentioned experiments mutually confirm and supplement each other.

Data on the concentration of an interplanetary ionized gas (particles possessing thermal energies) obtained from Lunik III at distances from the Earth of the order of $150000 \mathrm{~km}$ showed that, for such distances, the concentration of ions does not exceed a few particles per $\mathrm{cm}^{3}(6)$.

Before the beginning of flights of cosmic rockets it was impossible to make direct observations of solar corpuscular streams in interplanetary space because, when entering the geomagnetic field and upper atmosphere of the Earth, they become essentially changed.

At first, currents created by positive ions of solar corpuscular emission were registered in 1959 outside the Earth's magnetic field (at a distance of the order of $100000 \mathrm{~km}$. from the Earth) by means of three-electrode traps of charged particles placed on Soviet space probes. To the outer grids of the traps there were applied positive potentials (relative to the body of the rocket) retarding positive ions. Magnitudes of these potentials excluded the possibility of trapping ions with thermal velocities; that is why registered positive currents could be created only by ions of corpuscular streams $(3,6)$. A good correlation between values of registered currents and $K$-indices of geomagnetic variation, observed on the Earth, was found (6).

Three-electrode traps of charged particles orientated to the Sun were placed in the automatic interplanetary station launched on 12 February, towards Venus; one of those traps was specially designed for registration of the ionic component of solar corpuscular radiation. On 17 February 1961 during a period of communication with the automatic interplanetary station a passage of the station was registered through a noticeable flux of corpuscles (of order $10^{9}$ $\left.\mathrm{cm}^{-2} \mathrm{sec}^{-1}\right)$. Simultaneously a geomagnetic storm was observed (6).

In future such experiments will permit to fix quantitative relations between intensities of geomagnetic storms and intensities of solar corpuscular streams creating them.

Devices, so far applied in Soviet experiments, have only permitted a statement that registered streams of ions did not include ions with thermal velocities, and measuring density of a stream but not permitting the determination of energies of registered corpuscles. At present, devices have been prepared which will permit the determination of the energy spectrum of corpuscles coming into the traps.

2. The results of magnetic field measurements from the Venus Probe Automatic Interplanetary Station

On the Venus Probe three-component magnetometers, for measuring the field near the planet, and magnetic variometers, for in-flight measurements of relative variations of the field, were installed. The magnetic measurements were carried out by Sh. Dolginov, E. Eroshenko, L. Zhuzgov, N. Pushkov.

The magnetic variometer in use was a two-channel instrument with independent electronic elements and pick-ups. The pick-ups of the variometers were oriented in parallel, and thus measured the same component of the field.

To minimize the influence of the container on the instrumental readings, the pick-ups were fixed on an antenna at a distance of about 2 metres from the container.

The antenna was directed at an angle of the order of $43^{\circ}$ with respect to the normal to the solar cells. The sensitivity threshold of the variometers was 2 gammas, and the range of measurement was $5 \circ$ gammas. 
The variometer information was obtained during radio contacts on 12 February and 17 February 196r. Though the information obtained is comparatively limited (the time of information was 52 minutes), it is of undoubted interest since it throws light upon the multifarious features of a magnetic disturbance formation.

On 12 February the day was magnetically quiet according to data from magnetic observatories. Moderate magnetic disturbances took place on 13 February, 16 February and ${ }_{7} 7$ February; the last was most intense.

Fig. 4 presents the indications of the variometers when the Venus probe was in the vicinity of the Earth, but beyond its magnetic field, at a distance of $165000-175000 \mathrm{~km}$. Lower curves illustrate the field-variations on the Earth, according to data from a middle-latitude

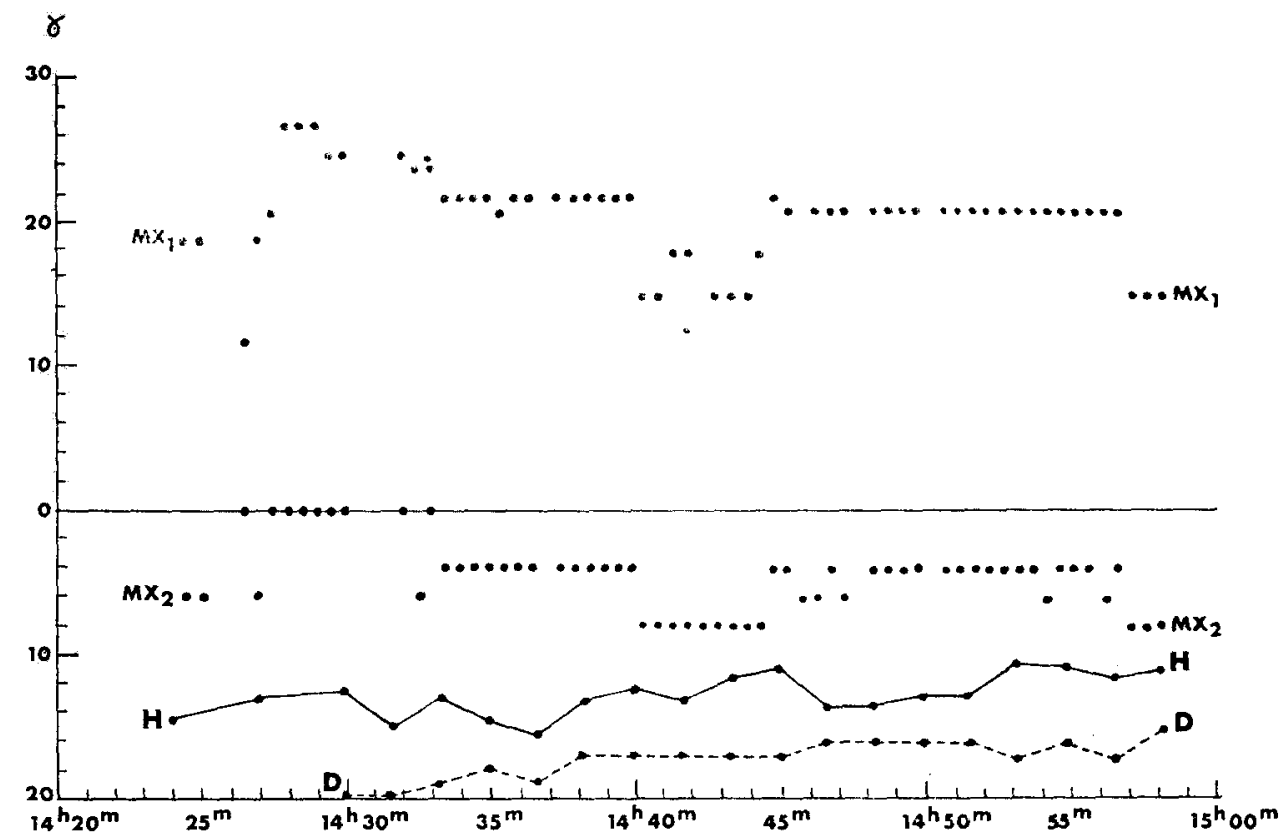

Fig. 4. Magnetometer indications of the Venus probe on 12 February $196 \mathrm{r}$; the dotted lines $\mathrm{MX}_{1}$ and $\mathrm{MX}_{2}$ indicate distances of about $170000 \mathrm{~km}$.

magnetic observatory. Both channels of the variometers revealed, in the main, co-ordinated changes about the average level within \pm 4 gammas. Characteristic changes, of 4 -minute duration, had the form of steps due to character of the information transmissions. The curve $\mathrm{H}$ plotted in the lower part of Fig. 4 shows changes within the same limits, but not synchronous.

Analysis of telemetry data of a number of instruments carried by the probe makes it possible to believe that these changes of the field are not connected with interferences from the probe, but reflect actual pictures of the fluctuations of the field in the closest vicinity of the Earth.

The next radio contact took place on 17 February at a distance of $1900000 \mathrm{~km}$ from the Earth. Fig. 5 shows the variometer indications and the field variations on the Earth at this time. The variometers during a 22 -minute radio contact show almost invariable readings. The Earth's magnetic field at this time revealed considerable changes which, according to data from the middle-latitude station, reached 20-25 gammas. Time-intervals before and after 
the time of observation on the Venus probe were also disturbed. It is impossible to expect similarity of the field-variations curves, but in this case the absence of synchronism in activity is revealed for the next time-intervals during the radio contact.

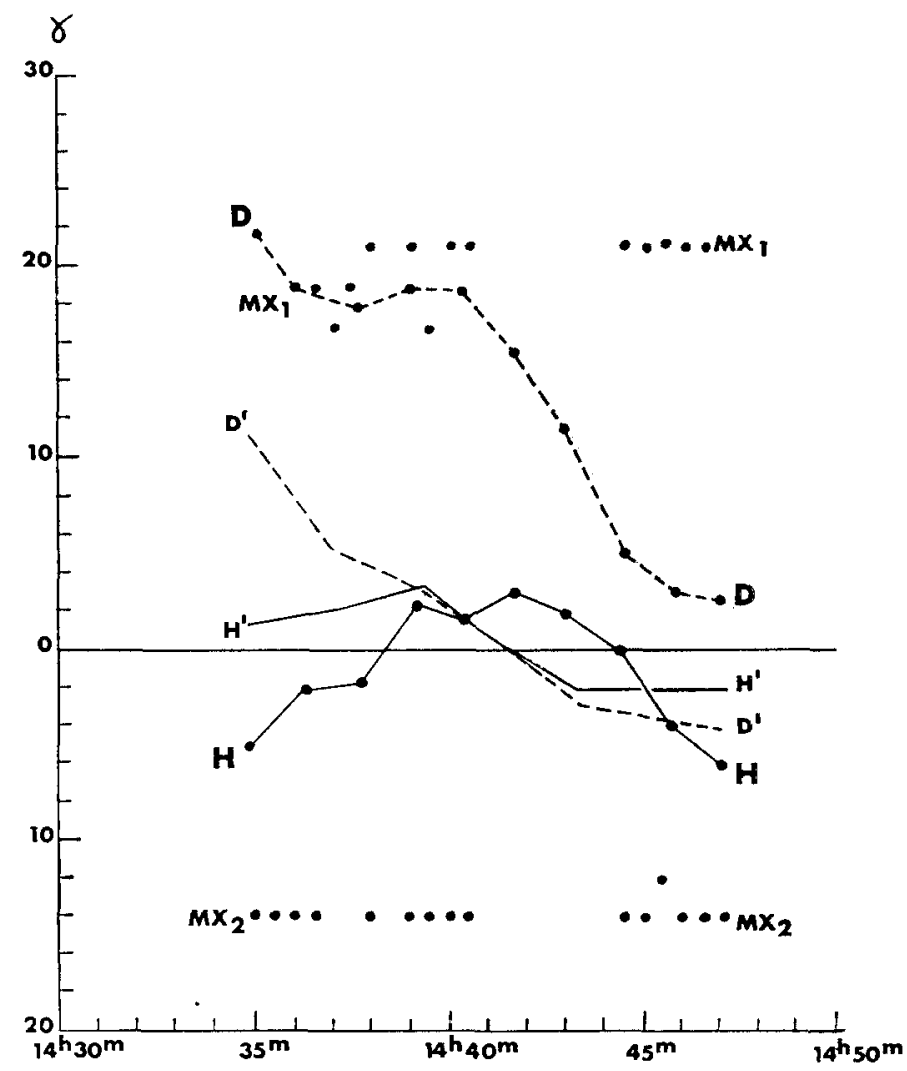

Fig. 5. Magnetometer indications of the Venus probe on I7 February 196r; the dotted lines $\mathrm{MX}_{1}$ and $\mathrm{MX}_{2}$ indicate distances of $\mathrm{I} 900000 \mathrm{~km}$.

The next important feature which should be mentioned in discussing the magnetic-measurement results is that other instruments of the probe, namely, detectors of charged particles, which also gave information during these radio contacts measuring low-energy corpuscular radiation, also revealed changes during the 17 February contact as compared to the 12 February contact. These changes can be explained if we suppose that on 17 February the probe was within a corpuscular stream. During the whole radio contact on 17 February the indications of the charged particle detectors did not change, as was the case with variometers.

The comparison of the average levels of the indications of the variometer channels on 12 and I7 February provide an indication of the magnitude of the magnetic field of the corpuscular stream revealed by the detectors. The average levels of counting by the $\mathrm{MX}_{1}$ variometer (see Figs. 4 and 5) were the same on 12 and 17 February. The level of the indications of the variometer $\left(\mathrm{MX}_{2}\right)$ on 12 and 17 February differs by 9 gammas. This indicates that the $\mathrm{MX}_{1}$ or the $\mathrm{MX}_{2}$ variometer experienced a drift of 9 gammas for five days. It is most likely that the $\mathrm{MX}_{1}$ variometer had no drift, and in this case the component of the stream field along the 
sensor axis was $\leqslant 2$ gammas. In another case it should be assumed that the component of the stream field was 9 gammas.

According to the theory of the Forbush effect the intensity of the stream magnetic field in the closest vicinity of the Earth can be obtained from the cosmic-rays variations. The ground data on the cosmic-ray neutron component variations are presented in Fig. 6. As evident from Fig. 6 a Forbush decrease at the Earth began six hours later and reached its maximum nine

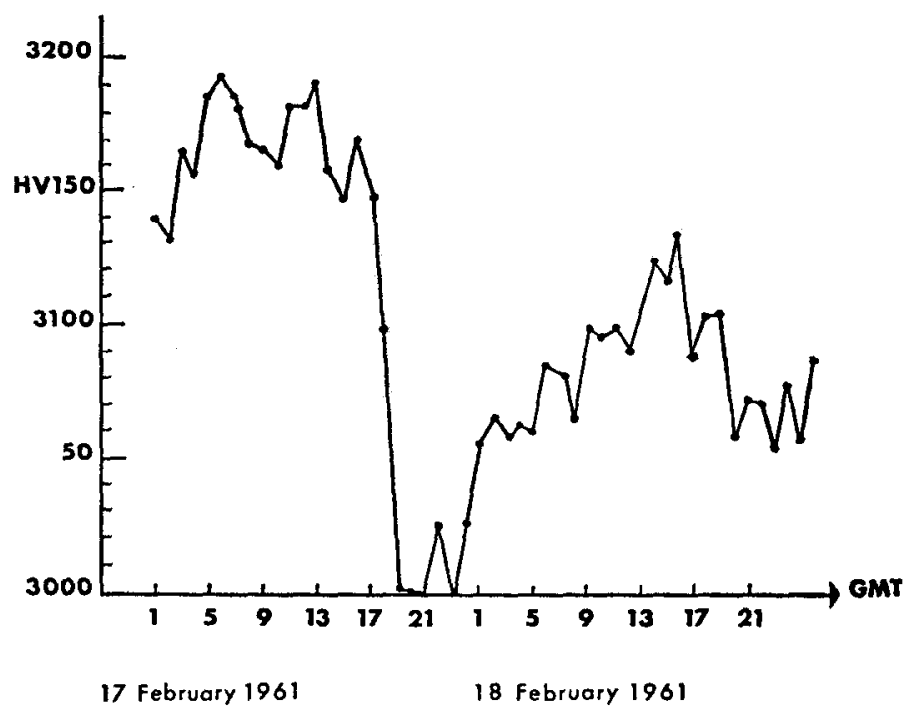

Fig. 6. Ground data (Jungfraujoch, Switzerland) on the variations of the cosmic ray neutron component. (Courtesy, Professor Dr H. F. Houtermans).

hours later than the observation made on the probe on 17 February 1961 . On the basis of the data presented the conclusion can be drawn that, at the moments of the observations of the Venus probe, the field of the stream was low, not only near the space probe but also in the vicinity of the Earth. Consequently the cause of geomagnetic disturbances at the Earth during radio contact with the Venus probe on 17 February was not frozen magnetic fields of the stream, but the other kind of transformations of the stream energy into magnetic energy within the Earth's magnetic field.

Comparing terrestrial data and the Venus probe data it is possible to conclude that in this experiment (unlike the Pioneer V experiment) no conditions existed when the disturbance of the magnetic field on the Earth is correlated with the disturbance of the field in interplanetary space.

\section{REFERENCES}

r. Krassovsky, V. I. Trans. $I A U$, то, 7 16, 1960.

2. Gringauz, K. I., Bezrukikh, V. V. and Ozerov, V. D. "Artificial Earth Satellites", issue $6,48,196 \mathrm{r}$.

3. Gringauz, K. I., Bezrukikh, V. V., Ozerov, V. D. and Rybinsky, R. E. C.R.Acad.Sci. U.R.S.S., 131, 1301, 1960.

4. Gringauz, K. I., Kurt, V. G., Moroz, V. I. and Schklovsky, I. S. C C.R.Acad.Sci.U.R.S.S., r32, $1062,1960$. 
5. Gringauz, K. I., Kurt, V. G., Moroz, V. I. and Schklovsky, I. S. $\quad A . Z h$., 37, 716, I960.

6. Gringauz, K. I. Space Research II. Proc. of the Second International Space Science Symposium, North Holland Publishing Co., Amsterdam, 196r.

7. Sonett, C. P., Smith, F. J., Coleman, P. J., Jr. and Judge, D. L. $\quad$ F.geophys.Res., 65, 1858 , 1960.

8. Gringauz, K. I. and Rytov, S. M. C.R.Acad.Sci.U.R.S.S., 135, 48, 1960.

\section{SCIENTIFIC RESULTS OF U.S. SPACE PROBE MEASUREMENTS}

\section{P. Sonett}

The survey nature of rocket study of cosmic hydromagnetics suggests that space-probe data be considered chronologically. In short, the experiments consist primarily of verification or denial of hitherto postulated models, substantiation of surface observations, and findings in some cases implying so great a deviation from current ideas that repetition of the experiment is suggested.

This summary dwells primarily upon magnetic field and plasma probe data with some attendant comments on cosmic ray results. To begin, we summarize the Pioneer I findings ( $\mathrm{I}$ ). These suggest (for II October 1958) a dipolar field from 3.7 to $7 R_{\mathrm{e}}\left(R_{\mathrm{e}}=\right.$ geocentric radii) and a magnetostatic compression at $12.4-{ }_{4} R_{\mathrm{e}}$ to twice nominal value. At $14 R_{\mathrm{e}}$ the field gradient steepens and the implication of a boundary suggests an interplanetary field less than $6 \gamma$. An elastic collisional process between a plasma wind and magnetosphere implied further an interplanetary proton density $\sim 1 \mathrm{I}^{2} \mathrm{~cm}^{-3}$ in qualitative agreement with later results. On the other hand, the finite amplitude waves noted at $12.3-14 R_{\mathrm{e}}$, presumably transported into the magnetosphere, might be expected to modify the momentum balance at the boundary (2), suggesting for this day a solar wind of $10^{-9}$ dynes $/ \mathrm{cm}^{2}$ pressure. These large waves, seen also on Pioneer V (3) and having an electron analogue on Pioneer IV (4), seem to fit the category of almost collisionless magnetic pulses propagating normal to the ambient field direction. The term "hyperwave" is suggested (5). Front thickness and downstream oscillations seem to satisfy the hypothesis of local acceleration in the magnetosphere (6). Such waves may be characteristic of colliding astrophysical plasmas.

The interior of the magnetosphere showed hydromagnetic wave activity having an energydensity $10^{-13}-10^{-12} \mathrm{ergs} / \mathrm{cm}^{3}$ over $\mathrm{O}-\mathrm{I} \mathrm{cps}$. The total magnetospheric hydromagnetic energy to $7 R_{\mathrm{e}}$ would then be $\sim$ I0 ${ }^{10} \mathrm{ergs}$ over $\mathrm{O}-\mathrm{I} \mathrm{cps}$. Details of the spectra and partial separation of modes is now being made ( 7$)$.

Explorer VI primary experiments were a triple coincidence $2 \pi$ cosmic-ray telescope (Simpson), an ion chamber-Geiger tube experiment (Winckler), and a search-coil magnetometer (Sonett). Useful life-time of the satellite was almost 2 months and allowed traversal of the Van Allen belts through two magnetic storms. Many results of Simpson's experiments have recently been published (8). We shall not consider the particle experiments here because of lack of space. A primary finding of the magnetometer was a storm-type disturbance field which implied a current source exterior to the peak of the second Van Allen belt (9). Another result was a persistent deviation in both the magnitude and direction of the geomagnetic field from dipolar on the anti-solar or night side at distances beyond $5 R_{\mathrm{e}}$ (Io). The gross configuration of currents associated with these disturbances has not been established. Presently, the two primary models appear to be a geomagnetic tail (II) or an annular current about the Earth. These data qualitatively fit either model though the former leaves unexplained the findings of Pioneer V, the variable latitude cosmic-ray knee, or the Soviet plasma probe results.

Wave phenomena observed by the Explorer VI magnetometer are presently being studied in conjunction with variations in scintillation counter rate (12). 
Simultaneity of magnetic storm events, Forbush decreases, and propagation of solar cosmic rays was studied during the lifetime of Pioneer V, launched I I March 1960. Instrumentation included a $2 \pi$ triple coincidence telescope operating sub-proportionally (Simpson); an ion chamber-Geiger tube combination utilizing a Neher-type integrating chamber (Winckler) and a search-coil magnetometer (Sonett). Five Forbush decreases at the position of the spacecraft correlated with magnetometer disturbances (13). The large storm sequence of 31 MarchI April r960 provided conclusive evidence of the interplanetary origin of galactic cosmic ray obscuration and of its magnetic nature. Fields of at least 20-50 $\gamma$ were noted during this time, having a radial extent estimated at 0.7 A.U. (14). There is now adequate experimental substance to support the mechanism of plasma-field ejection from the Sun with solar cosmic-ray trapping. Field-energy density, $E_{\mathrm{H}} \geqslant 10^{-8} \mathrm{ergs} / \mathrm{cm}^{3}$ required an ordered plasma-energy density $E_{\mathrm{P}}>$ $10^{-8} \mathrm{ergs} / \mathrm{cm}^{3}$ and the transit time from the Sun to vehicle implied a plasma density, $\rho>10 \mathrm{~cm}^{-3}$ but not necessarily much higher. If so, the flux of $E_{\mathrm{H}}$ is comparable to the gas-energy flux; the magnetic-energy flux is $\sim \mathrm{I}$ erg $/ \mathrm{cm}^{2} \mathrm{sec}$. During quiet times $(\sim 50 \%$ of the time of observation) a steady field of $\sim 2 \gamma$, making a large angle to the ecliptic, was observed. (15) A field of this configuration is most in accord with the interplanetary gas model of Chamberlain and Brandt and is incompatible with a wind of energy density comparable to or greater than $10^{-11} \mathrm{ergs} / \mathrm{cm}^{3}$. From Simpson's experiment the galactic cosmic-ray gradient in the radial direction was found to be $-15 \pm 20 \%$ (A.U. $^{-1}$ near Earth (13). At 6 Earth radii an anomaly consistent with Explorer VI was noted. (3) From 10-14 Earth radii fluctuations consistent with Pioneer I hyperwaves were noted.

Explorer X carried an Rb magnetometer, flux gates, a Sun scanner (Heppner), and a plasma probe (Bridge) into the anti-solar hemisphere to $37 R_{\mathrm{e}}$. A negative anomaly of $200-400 \gamma$ appeared at $\sim 2 R_{\mathrm{e}}$ qualitatively consistent with Soviet data (r6). No explanation is given. At $4.5 R_{\mathrm{e}}$ the field began to rotate in the same manner as Explorer VI. At $7 R_{e},|H|$ began to exceed nominal dipolar and by $2 \mathrm{I} \cdot 5 R_{\mathrm{e}}$ was still $32 \gamma$. Heppner et al. suggest a geomagnetic tail. Qualitative inconsistency with an annular current is not conclusive. Cosmic-ray latitude knee is, for example, explained by a ring current.

At $2 \mathrm{I} \cdot 5 R_{\mathrm{e}}$ penetration into a highly disturbed plasma occurred. Magnetic activity had been mild and continued to $37^{\cdot 2} R_{\mathrm{e}}$ when a sudden commencement at both vehicle and Earth surface occurred. The sudden commencement caused a spike in $|H|$ followed by a gradual rise in $|H|$. Field direction was highly variable outward from $2 \mathrm{x} \cdot 5 R_{\mathrm{e}}$ and did not seem to be influenced by the sudden commencement. In general, the field was highly agitated, suggesting waves and vortical motion. The appearance of boundary penetration at $2 \mathrm{I} \cdot 5 R_{\mathrm{e}}$ is strongly suggested and fits the $\sim{ }_{14} R_{\theta}$ boundary on the solar side established by Pioneers I and V.

Plasma activity close to the Earth consisted of passage through a cold medium $\left(1 \cdot 3-2.9 R_{\mathrm{e}}\right)(\mathbf{1} 7)$. From $2 \cdot 9-21 \cdot 5 R_{\mathrm{e}}$ plasma signal was not received, confirming proper photo-electric suppression in the equipment. This also bounds the plasma current to below $5 \times 10^{6} \mathrm{~cm}^{-2} \mathrm{sec}^{-1}$. (The instrument was biased to accept only protons with energy between vehicle potential and 2300 volts.) Beyond $21 \cdot 5 R_{\text {e }}$ plasma was seen most of the time $\left(4 \times 10^{8}\right.$ $\mathrm{cm}^{-2} \mathrm{sec}^{-1}$ at mean $E=500$ volts). The flux varied from threshold to $10^{9} \mathrm{~cm}^{-2} \mathrm{sec}^{-1}$, high values coinciding with weak field. Strong field in the anti-solar direction resulted in low plasma flux. Consideration of energy measurements yielded ion densities varying from $6-20 \mathrm{~cm}^{-3}$. Analysis of spacecraft spin-plasma intensity showed plasma-directed velocity to be away from the Sun. The sudden commencement observed on the magnetometer showed a protonenergy spectral shift to higher values.

A typical burst of data showed a region, perhaps $10^{10}-10^{11} \mathrm{~cm}$ where the magnetic fieldenergy density was $\sim 25^{\circ} \mathrm{ev} / \mathrm{cm}^{3}$, the ordered gas kinetic-energy density $\sim 5 \mathrm{kv} / \mathrm{cm}^{3}$, followed by a region perhaps $10^{11} \mathrm{~cm}$ in extent where $H \sim 20 \gamma\left(E_{\mathrm{H}} \sim 2 \mathrm{kv} / \mathrm{cm}^{3}\right)$ and 
$E_{\mathrm{P}} \sim \mathrm{I} \mathrm{kv} / \mathrm{cm}^{3}$. The former region implies a thermal energy component not exceeding $25^{\circ}$ $\mathrm{ev} / \mathrm{cm}^{3}$ or that $E$ disorder $\sim 0 . \mathrm{I} E$ order (18).

Detailed analysis of spin modulation of the plasma signal allowed extension of the energy spectrum accessible to higher values. The sudden commencement transit time from the Sun implied ro kv particles. There was no strong evidence for these and the hypothesis is raised of a wave propagation mechanism from Sun to Earth with attendant local acceleration (I8). The estimated particle volume densities for the two regions cited above are $n \sim 10 \mathrm{~cm}^{-3}$ and $n<0.2 \mathrm{~cm}^{-3}$ respectively. Basically, the plasma flux values are consistent with Soviet data. (19) The complexity of the magneto-hydrodynamics of the processes at play require further study of these data.

This summary is necessarily incomplete. Consideration of much of the data discussed is current and definitive interpretations are pending. The omission of Vanguard III is due to lack of adequate information by this author. Both spherical harmonic analysis and whistler data are being presently evaluated. We also have not included the discussion of the results of the Ionospheric Satellite, Explorer VIII.

Some accomplishments of this programme are:

I. establishment of the scale of Forbush decrease mechanisms;

2. discovery of a quiet interplanetary field;

3. determination of the interplanetary storm field magnitude;

4. simultaneous observation of solar cosmic rays in transit and on Earth;

5. discovery of large current systems in the exosphere;

6. observation of collisionless shocks in situ; and

7. determination of distances of the geomagnetic boundary.

\section{REFERENCES}

r. Sonett, C. P., Sims, A. R. and Smith, E. J. Rocket Surveys of Distant Magnetic Fields, Space Research, Ed. H. K. Kallman-Bijl, N. Holland Pub. Co., Amsterdam, 1960.

2. Sonett, C. P. Coupling of the Solar Wind and the Exosphere, Phys. Rev. Letters 5, 46, r 960 .

3. Coleman, P. J., Sonett, C. P., Judge, D. L. and Smith, E. J. Some Preliminary Results of the Pioneer V Magnetometer Experiment, F. geophys. Res. 65, 1856, 1960.

4. Van Allen, J. A. and Frank, L. A. Radiation Measurements to $658300 \mathrm{~km}$ with Pioneer IV, Nature, Lond. 184, 2 I9, 1959.

5. Davis, Leverett, Jr. Private communication.

6. Sonett, C. P. Hyperwaves, Shock-like Phenomena in the Outer Exosphere, Proc. of International Symposium on the Earth Storm and Cosmic Rays, Kyoto, Japan, I96r; F. phys. Soc. Fapan. 17, Suppl. A-II, p. 528.

7. Sonett, C. P., Sims, A. R. and Abrams, I. J. The Distant Geomagnetic Field I : Infinitesimal Waves in the Magnetosphere; in draft.

8. Fan, C. Y., Meyer, P. and Simpson, J. A. Dynamics and Structure of the Outer Radiation Belt, F. geophys. Res. 66, 2607, I96r.

9. Smith, E. J. and Sonett, C. P. Satellite Observations of the Distant Field During Magnetic Storms: Explorer VI, Proc. of the International Symposium on the Earth Storm and Cosmic Rays, Kyoto, Japan, 1961; Ұ.phys.Soc. Japan, 17, Suppl. AII.

ro. Sonett, C. P., Smith, E. J., Judge, D. L. and Coleman, P. J., Jr. Current Systems in the Vestigial Geomagnetic Field, Phys. Rev. Letters 4, r6r, I960.

Ir. Johnson, F. S. The Gross Character of the Geomagnetic Field in the Solar Wind, F. geophys. Res. 65, 3049, I960.

12. Farley, T. A. and Rosen, A. Charged Particle Variations in the Outer Van Allen Zone during a Geomagnetic Storm, 7 . geophys. Res. 65, 3494, I960.

13. Fan, C. Y., Meyer, P. and Simpson, J. A. Phys. Rev. Letters 5, 269 and 272, 1960. 
I4. Coleman, P. J., Jr., Sonett, C. P. and Leverett Davis, Jr. On the Interplanetary Magnetic Storm: Pioneer V, F. geophys. Res. 66, 2043, 1961.

15. Coleman, P. J., Jr., Leverett Davis, Jr. and Sonett, C. P. The Steady Component of the Interplanetary Magnetic Field, Phys. Rev. Letters 5, 43, 1960.

I6. Heppner, J. P., Ness, N. F., Skillman, T. L. and Scearce C. W. Magnetic Field Measurements with the Explorer X Satellite. Proc. International Symposium on the Earth Storm and Cosmic Rays, Kyoto, Japan 196I; Y. phys. Soc. Japan, 17, Suppl. A-II, p. 546.

r7. Bridge, H. S., Dilworth, C., Lazarus, A. J., Lyon, E. F., Rossi, B. and Scherb, F. Direct Observations of the Interplanetary Plasma, Proc. of International Symposium on the Earth Storm and Cosmic Rays, Kyoto, Japan, I96r.

18. Rossi, B. From remarks at Plenary Session on Explorer X. Proc. of International Symposium on the Earth Storm and Cosmic Rays, Kyoto, Japan, 196r. F. phys. Soc. Fapan, Suppl. AII.

19. Gringauz, Bezrukikh, Ozerov, ınd Rybinsky. C.R.Acad.Sci. U.R.S.S., 131, 1301, 1960. 